\title{
No One Likes to Be Second Choice*
}

\author{
Yair Antler \\ University of Essex
}

September 19, 2017

\begin{abstract}
A decision maker wishes to fill a vacancy with a fixed wage. Candidates who are more valuable to the decision maker are less likely to be available. The candidates suffer a disutility from filling the position when they are ranked low on the decision maker's preference list. However, the decision maker's preferences are his private information. Therefore, the candidates infer the decision maker's preference list from information revealed by the number of failed offers. We explore the adverse effect of the social component in the candidates' preferences on the decision maker's ability to recruit a suitable candidate.
\end{abstract}

Keywords: Social Motives; Interdependent Preferences; Hiring Procedures; Psychological Motives

\footnotetext{
*I am grateful to Rani Spiegler for his valuable guidance and support in this project. I acknowledge financial support from ERC grant no. 230251. I thank Benjamin Bachi, Kfir Eliaz, Alex Frug, Tobias Gamp, Jacob Glazer, Santiago Oliveros, Ariel Rubinstein, Heidi Thysen, and Asher Wolinsky for helpful comments. Correspondence: Yair Antler, University of Essex. E-mail: yair.an@gmail.com.
} 


\section{Introduction}

Salary is typically one of a combination of factors that affect a job candidate's decision to accept or reject a job offer. In addition to financial compensation, candidates often try to assess how much their new employer likes them and appreciates their skills. This endogenous assessment may signal the quality of the match, the likelihood of being respected and kindly treated in the new workplace, and the chances of early promotion or dismissal. Apart from its instrumental value as a proxy for these other dimensions of overall job satisfaction, a job candidate's desirability to his employer may be of fundamental value to the candidate due to social motives. The idea that people tend to like, think highly of, and want to interact with those who like them is supported by evidence from the social psychology literature dating back to Aronson and Worchel (1966). In the context of hiring, a job candidate who feels that he is not the employer's first choice for the position might refuse to fill a vacancy that he would otherwise be happy to fill.

Unlike wages, which are relatively easy to observe and verify, a potential employee is typically less informed about the extent to which his potential employer likes him and appreciates his skills. Nevertheless, his perceived desirability to the employer may affect the employee's decision to accept or reject a job offer. It is easier to identify the effects of desirability considerations on this decision in labour markets in which wages are highly regulated (e.g., public sector positions, some European academic labour markets) and job candidates cannot make inferences from the offered compensation. Instead, candidates form their assessments about the extent to which their potential employer values them based on observables such as the time between the posting of a vacancy and the time a recruiter contacts them to schedule an interview, the order in which candidates are interviewed, and how many candidates are considered for the position. The job candidates' inferences make the potential employer's problem a strategic one. In what order should the employer approach the candidates in the presence of social motives? Can he benefit from withholding information from the candidates or from delegating his decision to an uninformed party?

We study the effects of the above strategic elements on an employer's ability to appoint a suitable candidate. In our model, there is a decision maker (DM) who wishes to fill a vacancy with an exogenous wage. The DM approaches potential candidates sequentially. Each candidate is characterised by his availability and his value to the DM. The former (latter) aspect is the candidate's (DM's) private information. Value and availability are negatively correlated, reflecting that better candidates are typically 
more valuable in the market. The DM can make offers to all candidates and there is no cost of delay. The candidates in our model suffer a disutility from accepting an offer if they are ranked low on the DM's preference list. This complicates the DM's scheduling problem since candidates may infer their relative position on his preference list from the order in which he approaches them or from the number of failed offers (the candidates' beliefs about their ranking are also affected by their outside options, i.e., their availability).

Without taking social motives into account, the DM will approach the candidates according to his preference ordering (i.e., according to the candidates' values). To illustrate the trade-off between value and availability that emerges endogenously in our model, consider the above scheduling strategy and suppose that candidates reject the DM's offers if they are unavailable or if they think that they are not ranked first on his preference list. If the first candidate who is approached by the DM is unavailable, the DM will make a job offer to the next candidate on his preference list. The latter candidate can infer from the timing of the offer that he is ranked second and, therefore, he will reject the DM's offer. If, however, the DM takes this into account and believes that his favourite candidate is unlikely to be available, he might give up on the above scheduling strategy in favour of first approaching a candidate who is ranked lower on his preference list but is more likely to be available. However, a different scheduling strategy has implications on the inferences made by the candidates in equilibrium. For instance, if the DM approaches lower-ranked candidates first, a candidate who is approached first must infer that he is not ranked first on the DM's preference list and reject the DM's offer. This complicates the DM's problem and further exacerbates the adverse effect of the social motives.

Our main result is that under a mild condition on the distribution of candidates' types, when the number of candidates is large, in every symmetric Nash equilibrium the DM is unable to fill the vacancy. This result implies that having many potential candidates makes it hard for the DM to recruit a candidate, and contrasts with a result that without the social component, the DM is always better off when the number of candidates is increased.

We examine different mechanisms that allow the DM to control the information his offers reveal to the candidates. In particular, we compare between the case in which the DM makes his offers publicly and the case in which he makes them privately such that candidates cannot observe the number of failed offers. While the DM is better off making his offers privately when the candidates' social motives are weak, he is better off making them publicly when their social motives are strong. 
The paper proceeds as follows. Section 2 presents the model. Section 3 provides an illustrative example and Section 4 presents the general analysis. Section 5 compares the cases of private and public offers. Section 6 covers the related literature and Section 7 concludes by discussing a few extensions and implications. Proofs that do not appear in the main text are to be found in the Appendix.

\section{The Model}

Let $I=\{1, \ldots, n\}$ be a set of candidates and suppose that a decision maker (DM) wishes to appoint one and only one of these candidates to a position. Let $v_{i}$ denote the DM's value from appointing candidate $i \in I$ to the position. We assume that $v_{1}, \ldots, v_{n}$ are $n$ random variables drawn independently from a density $f>0$ on $\left[v_{l}, v_{h}\right], v_{h}>v_{l} \geq 0$. The values' realisations are the DM's private information while $f$ is commonly known. For each candidate $i \in I, x_{i}=\left|\left\{j \in I \mid v_{j}>v_{i}\right\}\right|$ is the number of candidates who have a higher value than $i$. Let $k \in\{1, \ldots, n-1\}$ and let $c>1$. The utility that candidate $i \in I$ derives from accepting the DM's offer is 1 if $x_{i}<k$ and $1-c$ otherwise. ${ }^{1}$ The utility derived by each candidate $i \in I$ from rejecting the DM's offer is 0 .

Let $s_{i} \in\{0,1\}$ denote candidate $i$ 's availability, where $s_{i}=1$ (respectively, $s_{i}=0$ ) means that $i$ is available (respectively, unavailable). Define $p\left(v_{i}\right):=\operatorname{Pr}\left(s_{i}=1 \mid v_{i}\right)$. We assume that $p:\left[v_{l}, v_{h}\right] \rightarrow[0,1]$ is continuous and nonincreasing in $v$. That is, there is a negative correlation between each candidate's value to the DM and the probability that the candidate is available (better candidates are less likely to be available). Each candidate's availability is his private information while $p$ is commonly known. One can interpret $s_{i}$ as agent $i$ 's outside option, such that unavailable (respectively, available) candidates are simply candidates with a good (respectively, bad) outside option.

Denote the set of rounds by $N=\{1, \ldots, n\}$. In each round $t \in N$, the DM makes a binding offer to one candidate. If that candidate accepts, then he fills the position and the game ends. If he rejects the offer, then at round $t+1$ the DM makes an offer to a candidate who has not rejected an offer previously. If by the end of round $n$ the DM's offers have all been rejected, the game ends and his payoff is 0 . Denote the set of permutations of $N$ by $\Pi_{N}$. A pure strategy for the DM is a permutation of $N$ for each realisation $\left(v_{1}, \ldots, v_{n}\right)$. In other words, a pure strategy $a:\left[v_{l}, v_{h}\right]^{n} \rightarrow \Pi_{N}$ maps realisations of candidates' values into permutations of the $n$ rounds. A mixed

\footnotetext{
${ }^{1}$ One can interpret $c$ as the intensity of the candidates' social preferences. At the end of this section we analyse a benchmark model in which the candidates' preferences do not contain a social component (i.e., $c=0)$. We do not address the case of $c \in(0,1)$ since its analysis is similar to the case of $c=0$.
} 
strategy $\alpha:\left[v_{l}, v_{h}\right]^{n} \rightarrow \Delta\left(\Pi_{N}\right)$ maps realisations of candidates' values into probability distributions over permutations of $N$. For each realisation $\left(v_{1}, \ldots, v_{n}\right)$, let $\alpha\left(\pi \mid v_{1}, \ldots, v_{n}\right)$ denote the probability that the mixed strategy $\alpha$ maps the realisation $\left(v_{1}, \ldots, v_{n}\right)$ to the permutation $\pi$. For each realisation $\left(v_{1}, \ldots, v_{n}\right)$, permutation $\pi$, and round $t \in N$, we denote the candidate who receives an offer in round $t$ according to $\pi$ by $\pi_{t}\left(v_{1}, \ldots, v_{n}\right)$.

We assume that a candidate who is unavailable rejects the DM's offer. For each available candidate $i \in I$, a behavioural strategy $\sigma_{i}: N \rightarrow[0,1]$ is the probability of accepting an offer conditional on the round in which it is made. We denote the probability that an available candidate $i$ accepts an offer in round $t$ by $\sigma_{i}^{t}$. We say that the DM's strategy a treats the candidates in a symmetric manner if it is not conditioned on the candidates' labels. Formally, a treats the candidates in a symmetric manner if $a$ maps candidate $j$ to round $h$ under $\left(v_{1}, \ldots, v_{n}\right)$ if and only if $a$ maps candidate $\pi_{i}$ to round $h$ under $\left(v_{\pi_{1}}, \ldots, v_{\pi_{n}}\right)$ whenever both $v_{i} \neq v_{j}$ for each $v_{i}, v_{j} \in\left(v_{1}, \ldots, v_{n}\right)$, and $\left(v_{\pi_{1}}, \ldots, v_{\pi_{n}}\right)$ is a permutation of $\left(v_{1}, \ldots, v_{n}\right)$ such that $\pi_{i}=j$. Mixed strategies that treat the candidates in a symmetric manner are defined analogously.

We restrict our attention to Nash equilibria in which the DM's strategy treats the candidates in a symmetric manner and the candidates use symmetric strategies (that is, $\sigma_{i}^{t}=\sigma_{j}^{t}$ for each $i, j \in I$ and $\left.t \in N\right)$. We refer to this solution concept as a symmetric Nash equilibrium (SNE). Since the candidates' strategies are symmetric we omit the subscript from the description of their strategies and use $\sigma^{t}$ to denote the probability that an available candidate who receives an offer in round $t \in N$ accepts it.

\section{Discussion: Modelling Assumptions}

\section{Preferences versus behaviour}

In the context of this paper, there are two types of social motives that we can consider: the candidates may care about the order in which they are offered a position (e.g., whether they receive an offer first or not) or about their relative ranking in the DM's preference list. Both motives can follow from the existence of an audience of outside observers who make inferences about the candidates' ranking in the DM's preference list (correct inferences in the case of the latter motive and possibly incorrect inferences in the case of the former motive). In both cases, we can interpret the disutility derived by a candidate as a disutility from working for an employer who made him look bad in public. We focus on the latter motive, which may also result from the other considerations, elaborated in the introduction. If, in addition to the motive which we focus on, we were to incorporate the former motive into our model (e.g., by assuming that the candidates 
obtain a disutility if they accept offers in a late round), it would only exacerbate the adverse effect of the social component in the model and strengthen our results.

\section{Symmetry}

Restricting our attention to equilibria in which the DM's strategy treats the candidates in a symmetric manner enables us to obtain full support and thereby avoid making assumptions about the candidates' beliefs off the equilibrium path. To see this, observe that since the candidates' values are i.i.d., there is a strictly positive probability for each permutation of the DM's offers.

We also assume that the candidates use symmetric strategies. Let us consider the main effect of this assumption. When the candidates' strategies are symmetric, a candidate who receives an offer in round $t$ does not care who are the $t-1$ candidates who rejected the DM's offers in rounds $1, \ldots, t-1$. This information affects the candidate's beliefs about his relative ranking only when the other candidates' strategies differ. If we assume that an available candidate who receives an offer knows only the time $t \in N$ in which he is approached rather than the complete history of offers made by the DM, then we can relax the assumption that the candidates use symmetric strategies, without changing any of the paper's results. Under the symmetry assumption, there is no need to distinguish between the case in which an agent knows only the time in which he receives an offer and the case in which he knows the complete history. We keep the symmetry assumption since it significantly simplifies the exposition.

\section{A Benchmark}

Before proceeding to the analysis, we present a simple result to serve as a benchmark. It establishes that in the absence of social motives $(c=0)$, in the unique SNE, the DM approaches the candidates in the order of their values (i.e., he approaches his most-valued candidate first, his second-most-valued candidate second, and so on).

Claim 1 Suppose that $c=0$. Then, in the unique SNE, the DM approaches the candidates in the order of their values.

Proof. Since $c=0$, each available candidate accepts an offer regardless of the round in which it is made. Therefore, approaching the candidates in the order of their values first-order stochastically dominates any other strategy. 
This result follows directly from the fact that there is no cost of delay because available candidates always accept the DM's offers. That is, there is no cost in making offers to candidates who are less likely to be available. However, once candidates have social preferences, their likelihood of being available plays an important role in the DM's strategic considerations.

\section{An Illustrative Example}

Let us assume that there are two candidates and that each of them prefers to fill the position to not doing so if and only if he believes that he is ranked first on the DM's preference list with a probability of more than $\frac{1}{2}$. The latter assumption is equivalent to assuming that $c=2$. To focus on the main intuitions of the model, we simplify it to the bare minimum by assuming that the candidates' values are drawn from the uniform distribution on $\left[\frac{1}{2}, 1\right]$ and that $p(v)=1-v$.

In what order should the DM approach the candidates? A natural scheduling strategy is to approach the candidate whom he likes best first. The problem with this strategy is that the candidates infer their exact ranking from the timing of the DM's offers. As a result, the candidate who receives the second offer rejects it. Conditional on having his second offer rejected, the DM will prefer to make his first offer to the candidate for whom $p(v) v$ is maximised. However, since $p(v) v=(1-v) v$ is decreasing in $v$, the candidate for whom $p(v) v$ is maximised is the candidate who is least valued by the DM. Therefore, the strategy of always approaching the most-preferred candidate first cannot be a part of an SNE.

The social motives in this model have an adverse effect as candidates who believe that the DM likes others better than he likes them reject his offers. How strong is this effect? Can it be the case that, regardless of their availability, both candidates reject the DM's offers in an SNE? Such an SNE would be inefficient as even when the DM's most-preferred candidate is available, he will refuse to fill the position. It turns out that such an equilibrium exists.

Claim 2 There exists an SNE in which the DM randomises uniformly between permutations and the candidates reject both of his offers (i.e., $\sigma^{1}=\sigma^{2}=0$ ).

Let us consider the information that is (not) revealed to the candidates in this SNE. Since the DM randomises uniformly between the two permutations, the candidates learn nothing from the order in which the DM approaches them. Since $p(v)$ is decreasing in $v$, conditional on being available, a candidate's interim belief is that 
he is ranked first with a probability of less $\operatorname{than}^{2} \frac{1}{2}$. Therefore, the candidate who is approached first rejects the DM's offer regardless of his availability. As a result, the candidate who receives the offer second learns nothing from the fact that the first offer was rejected and rejects the DM's offer as well.

The SNE that we have described results in the worst possible outcome for the DM. In our setting, there may exist multiple equilibria so that it makes sense to examine the DM's payoff in other SNEs. The following claim establishes that in all of these SNEs, the DM cannot recruit any candidate.

Claim 3 In every SNE, all of the DM's offers are rejected (regardless of the candidates' availability).

First, let us understand why an SNE in which offers are accepted with some probability in one round and always rejected in the other round (e.g., an SNE in which $\sigma^{1}>0$ and $\sigma^{2}=0$ ) cannot exist. If there were an SNE in which candidates only accept offers made in the first round, then the DM would settle the trade-off between value and availability in favour of first approaching the candidate for whom $p(v) v=(1-v) v$ is maximised. However, this candidate is the one who is more likely to be available and less valued by the DM. As a result, a candidate who receives the first offer must infer that he is ranked second on the DM's preference list and reject his offer.

Second, we provide the intuition for the nonexistence of an SNE in which offers are accepted with strictly positive probability in both rounds (i.e., an SNE in which $\sigma^{1}>0$ and $\sigma^{2}>0$ ). Consider an available candidate $i$ and recall that because of the negative correlation between value and availability, candidate $i$ 's interim belief is $\operatorname{Pr}\left\{v_{i}>v_{j} \mid s_{i}=1\right\}<\frac{1}{2}$. For candidate $i$ to accept an offer, he must believe that he is ranked first with a probability of at least $\frac{1}{2}$. Hence, for $i$ to accept the DM's offer in the first round, he must infer some positive information about his ranking from the fact that the DM approached him first. However, if $i$ is ranked first on the DM's preference list, then $j$ is ranked second on that list, and vice versa. Therefore, when $i$ makes a positive inference about his ranking from the fact that he receives the first offer, this necessarily implies that candidate $j$ makes a negative inference about his own ranking from the fact that $i$ is approached first. Thus, accepting the DM's offer cannot be a best response for both $i$ and $j$.

In equilibrium, the DM's strategy is optimal with respect to his preferences. This creates a problem for the DM as he cannot control the information his strategy reveals to the candidates. In the example, $p(v) v$ is decreasing in $v$ so that candidates make

\footnotetext{
${ }^{2}$ The exact probability is calculated in the proof of Claim 2.
} 
negative inferences about their ranking when they receive an offer and this leads them to reject it. If the DM could instead commit to a suboptimal strategy, for example, to making the first offer to the candidate who is ranked first on his preference list, the problem would be partially solved. In such a case, an available candidate who receives the first offer will infer that he is ranked first on the DM's preference list and accept it.

At this point, we would like to mention that there exist nonsymmetric Nash equilibria in this setting. ${ }^{3}$ In the appendix, we provide an example of such a Nash equilibrium and generalise Claim 3 by showing that in every Nash equilibrium all of the DM's offers are rejected.

\section{Analysis}

In the example, we made several simplifying assumptions that helped us present the main ideas underlying the model. For example, the assumption that $p(v) v$ is decreasing in $v$ implies that when there is only one "effective" offer to make, for any realisation $\left(v_{1}, v_{2}\right)$, the DM makes that offer to the least-valued candidate. When $p(v) v$ is nonmonotone in $v$ and the DM has one effective offer to make, he makes this offer to the most-valued candidate in some realisations and to the least-valued one in others. In this section, we show that for large values of $n$, the same logic leads to unravelling even when we relax the assumption that $p(v) v$ is decreasing in $v$.

Another key assumption of the example is that $n=2$. This allows us to calculate the candidates' beliefs by hand and show that an SNE in which offers are accepted with a strictly positive probability in both rounds cannot exist. When $n$ is large, the difference between the belief of a candidate who receives the first offer and the belief of a candidate who has observed many failed offers can be so great that, a priori, it is not clear if the argument presented in the example will work.

In this section, we relax the above limitations and focus on the adverse effect of social motives on the DM's ability to hire a suitable candidate. The first proposition provides a tight sufficient condition for the existence of an SNE that induces the worst outcome possible for the DM.

Proposition 1 Suppose that $\frac{k}{n} \leq \frac{c-1}{c}$. For any $f$ and $p(v)$, a profile of strategies in which $\sigma^{1}=\ldots=\sigma^{n}=0$ and the DM mixes uniformly among all permutations is an SNE.

\footnotetext{
${ }^{3}$ I thank an anonymous referee for this point.
} 
The condition in Proposition 1 implies that a candidate who believes that his likelihood of being among the top $k$ candidates is "lower than average" will not be willing to accept an offer. Without receiving any information, no candidate believes that he is better than the average candidate. Therefore, all of the candidates reject the DM's offers. Although it is only a sufficient condition for the existence of such an SNE, it is tight in the sense that if $\frac{k}{n}>\frac{c-1}{c}$, then one can find a distribution of candidates' values and a function $p(v)$ such that the DM's offers are accepted with strictly positive probability in each SNE.

The next proposition is the paper's main result. We show that under a mild assumption about the distribution of types, the DM is unable to hire anyone in any SNE. This implies that in contrast to a setting with no social preferences, having many potential candidates makes it harder for the DM to appoint a candidate.

Proposition 2 Fix $k$, let $v^{\star} \in\left(v_{l}, v_{h}\right)$, and suppose that $v p(v)$ is strictly decreasing in $v$ for $v>v^{\star}$. There exists a number $n^{\star}$ such that for each $n>n^{\star}$, in every SNE, all of the DM's offers are rejected (i.e., $\sigma^{1}=\ldots=\sigma^{n}=0$ ).

Proposition 2 establishes that increasing the number of candidates may decrease the DM's highest SNE payoff. Recall that in the absence of social preferences, increasing the number of candidates strictly increases the DM's payoff. This effect is generated because it becomes harder for the DM to signal his preferences to the candidates whom he likes most as the number of candidates increases.

To provide a clear intuition for the proof, suppose for a moment that the DM has only one offer he can make. He will make that offer to the candidate $i$ with the maximal $p\left(v_{i}\right) v_{i}$. Candidate $i$ need not be the candidate with the maximal value for the DM. By our assumption that $v p(v)$ is strictly decreasing in $v$ for $v>v^{\star}$, if the DM faces a realisation in which there are $k^{\prime}>k$ candidates with values greater than $v^{\star}$, then he will make his only offer to a candidate who is not among his $k$-most-valued candidates (i.e., to a candidate $i$ such that $x_{i} \geq k$ ).

The above argument can be applied to the last round in which the DM's offer might be accepted in an SNE. Consider an SNE in which the DM's offers are accepted by available candidates in some rounds. Denote the set of those rounds by $D_{a}$ and the set of the rounds in which the DM's offers are rejected in this SNE by $D_{r}$. The optimality of the DM's strategy implies that the candidate who is scheduled to receive an offer in the last round in $D_{a}$ has a higher $p(v) v$ than each candidate who is scheduled to receive offers in $D_{r}$. By our assumption that $v p(v)$ is strictly decreasing in $v$ for $v>v^{\star}$, if the DM faces a realisation in which there are more than $\left|D_{a}\right|+k$ candidates with values 
greater than $v^{\star}$, then in the last round of $D_{a}$, he will make an offer to a candidate who is not among his $k$-most-valued candidates. When $n$ is large relative to $\left|D_{a}\right|$, the probability of such a realisation is high. This leads to a contradiction to the existence of an SNE in which the DM's offers are accepted since it is not a best response for a candidate to accept an offer in the last round in which he is supposed to accept it.

The argument presented above can be applied only when $n$ is large relative to the number of rounds in which offers are accepted with positive probability. When we increase the number of candidates $n$, the set of SNEs changes, such that, potentially, the number of rounds in which the DM's offers are accepted with positive probability in an SNE may increase when $n$ rises. A key aspect of the proof is to show that for any $n$, the number of rounds in which the DM's offers are accepted with positive probability in any SNE is bounded from above by some finite number $T$. In other words, for any $n$, the DM has at most $T$ opportunities to recruit a candidate. This technical result implies that we can treat this game as one with a finite number of offers and apply the argument presented in the previous paragraph when $n$ is large.

The optimality of the DM's strategy with respect to his preferences implies that the DM cannot fully control the information he reveals to the candidates. Since the candidates make negative inferences about their ranking from the DM's scheduling strategy, it makes sense to examine whether hiding his strategy can help the DM to overcome the negative effect of the candidates' social motives.

\section{Private Offers}

The model assumes that each candidate knows in which round he received an offer. We now identify the conditions under which the DM is better off hiding this information from the candidates. To do so, we examine an alternative model in which the offers are made privately. Since the offers are private, a candidate can observe only whether he received an offer or not. As a result, we need to redefine the candidates' strategies. A behavioural strategy $\gamma_{i} \in[0,1]$ for candidate $i$ is the probability that $i$ accepts the DM's offer in the case where he is available and receives one. Note that $\gamma_{i}$ is not conditioned on the round in which it is made since this is unobserved by $i$. As before, we restrict our attention to SNEs and omit the subscript $i$ such that $\gamma$ is the probability that an available candidate accepts an offer.

The fact that a candidate receives an offer is a positive signal about his relative ranking. To see this, note that when offers are made privately, there is no cost in approaching an unavailable candidate. Therefore, it is a dominant strategy for the DM 
to approach the candidates in the order of their values. It follows that the probability of receiving an offer is higher for the candidates who are ranked higher on the DM's preference list.

The natural question to ask is which of the two offer regimes, private or public, induces a greater payoff for the DM. In the next proposition, we shed light on this matter and connect it to the intensity of the social motives. We restrict our attention to the natural case of $k=1$ and show that if $c$ is large (small), then the private offers regime induces a lower (higher) payoff for the DM than the highest SNE payoff under the public offers regime.

Proposition 3 Let $k=1$ and suppose that $p\left(v_{h}\right)<1$. There exists a number $c^{\star}$ such that for every $c<c^{\star}\left(c>c^{\star}\right)$, the DM's highest SNE payoff under the public offers regime is weakly lower (weakly higher) than his highest SNE payoff under the private offers regime.

When the social component $c$ is relatively low, the private offers regime induces a strictly higher SNE payoff for the DM, compared to his payoff under the public-offers regime. This is because under the public offers regime, if the DM approaches the candidates according to their values, some of them will infer that they are not among the $k$-most-valued candidates and, therefore, reject his offers. Under the private offers regime, a candidate who receives an offer receives a positive signal about being one of the $k$-most-valued candidates. Thus, the DM can make all of the candidates believe that they are among the $k$-most-valued candidates with a probability of more than $\frac{c-1}{c}$.

We established that if $c$ is large, then the highest SNE payoff under public offers is weakly greater than the one under private offers. In fact, the highest SNE payoff under the public offers regime can be strictly greater than the latter. To see this, consider the case of $k=1, v_{l}>1$, and $p(v)=\frac{1}{v}$. If $c$ is sufficiently large, the highest SNE payoff under the private offers regime is 0. Under the public offers regime, there exists an SNE in which the DM approaches his most-preferred candidate first (for every realisation), $\sigma^{1}=1$, and $\sigma^{t}=0$ for $t>1$.

The private offers regime induces a positive signal about the relative ranking of candidates who receive offers. Under the public offers regime, some of the candidates receive positive signals about their ranking when they are made an offer, while others view the offer they receive as a negative signal about their ranking. While under private offers more candidates receive positive signals about their ranking, these signals are not sufficiently strong. Under public offers some candidates may receive stronger positive signals, but others make negative inferences about their ranking from the information 
that they receive. When $c$ is low, the intensity of the signal is not important and the DM is better off making offers privately. When $c$ is high, the intensity of the signal matters and the DM can be strictly better off by making his offers publicly.

\section{Related Literature}

The model is related to the literature on interdependent preferences. Gul and Pesendorfer (2016) provide a framework for studying interdependent preferences with incomplete information. Avery and Levin (2010) study early admissions at selective colleges using a model in which there are interdependencies between the preferences of the colleges and those of the students. In their model, early admissions help students to signal their preferences for one college or another. In the context of interdependent preferences, we are unaware of any other models in which agents' preferences are inferred from the timing of their actions.

Postlewaite (1998) provides an insightful discussion about incorporating concerns for relative ranking into economic models. Antler (2015) studies the effect of social preferences on the design of mechanisms for two-sided matching in a model of complete information. The conventional two-sided matching problem is extended by allowing the agents' preferences to depend on the endogenous messages of agents on the other side of the market and their interpretation by an audience of outside observers.

Our paper is related to the literature about contracting with externalities. In Jehiel and Moldovanu (1995), there is a random order of meetings and exogenous negative externalities that may lead to a delay in negotiations. In our model, the order is not random, the externalities are endogenous, there is no delay, but there may be unravelling. Jehiel, Moldovanu, and Stacchetti (1996) take a mechanism design approach to analyse a setting with exogenous contracting externalities.

Evidence from other fields: Social/psychological motives

Bretz, Gerhart, and Rynes (1991) interviewed university graduates about their job search and choice decisions. They found that the vast majority of the subjects were approached by some organisations late in the recruitment process and over 70 percent of them attributed these delays to "personal rejection or relegation to second-choice status." The majority of those subjects stated that delays negatively affected their willingness to accept job offers. 
Aronson and Worchel (1966), Curtis and Kim (1986), and Condon and Crano (1988) presented subjects with false evidence that other subjects had indicated that they liked them or enjoyed interacting with them. As a result, the liked subjects favoured these other subjects and gave them more positive evaluations. In the context of hiring, Alderfer and McCord (1970) found a high correlation between job applicants' expectations of receiving a job offer from a potential employer and these applicants' willingness to accept such an offer.

\section{Concluding Remarks}

We presented a model in which social motives affect overall job satisfaction and, as a result, an employer who wants to fill a vacancy must actively trade off between the candidates' value and availability. These social motives make the costs of pursuing the employer's top choice endogenous. Although our setting resembles a hiring situation, similar social motives are also prevalent in the contexts of team formation and romantic relationships.

For the DM, the problem is that some of the candidates who draw negative inferences about their relative value are "insulted" and reject his offers. Since the DM will inevitably "insult" some of the candidates, he prefers to insult those who are less likely to accept his offers in the first place, and this only exacerbates the adverse effect of the social motives. Since the DM targets candidates who are more likely to be available, candidates who receive an offer in a round in which they are supposed to accept it infer that they are unlikely to be the most-preferred ones. The candidates' inferences make the strategic choice of the DM significantly more complicated and create inefficiencies as efficient matches do not occur in equilibrium.

The model captures considerations that play a significant role in many real-world situations. For example, a job-market candidate will prefer going to an academic department that likes him better. In the real world, additional considerations might come into play but as long as wages are not fully adjustable, social motives will have an effect on the efficiency of the outcome of the recruiting process. Prominent examples where wages are not fully adjustable are public positions (e.g., the chair of a public

committee or a central bank) and senior functionaries in international institutions (e.g., the IMF).

We have shown that making offers privately cannot help the DM when the social motives are strong. We now examine a few other variants of the model and compare 
the DM's highest SNE payoff to his highest SNE payoff in the baseline model.

\section{Reducing the number of offers}

A natural question to ask is whether the DM can gain by committing to make a lower number of offers (fewer than $n$ offers). Such a commitment cannot benefit the DM when the number of candidates is large. To see this, suppose that the DM commits ex ante to make only $y<n-\frac{c k}{c-1}$ offers and consider an SNE in this game. In the game without commitment there is an SNE in which the DM and the candidates behave as in the game with commitment in the first $y$ rounds. From round $y+1$ onward, all of the DM's offers are rejected and he randomises uniformly among the permutations.

\section{Reference groups}

Individuals often compare themselves to people whose characteristics and abilities are similar to theirs. For example, in the context of the present paper, it is plausible that a candidate who finds it insulting to be "second choice" to a candidate similar to him (i.e., of his calibre) may not be so offended if someone who is a "superstar" is preferred to him. We now modify the model so that each candidate cares only about his ranking within his reference group. Thus, in the "superstar" example, non-superstars care only about their ranking among other non-superstars. Intuitively, superstars are far less likely to be available than non-superstars. Therefore, a candidate's lack of availability implies that he is more likely to be a superstar. As a rough approximation of this situation, suppose that each candidate's reference group coincides with the set of available candidates (i.e., each available candidate prefers to accept the DM's offer over rejecting if and only if he is among the $k$-most-valued available candidates). This would fit the "superstar" story in the extreme case in which the distribution over types and availability is such that lack of availability implies that the candidate is a superstar with a very high probability.

We now show that in this extreme example, the inefficiency problem disappears as there exists an SNE in which the DM always recruits the most-valued available candidate. ${ }^{4}$ Consider a profile of strategies in which every available candidate always accepts the DM's offers and the DM approaches the candidates in the order of their values. Given this profile, a candidate $i$ who receives an offer in round $t$ infers that

\footnotetext{
${ }^{4}$ Other SNEs may also exist. In particular, if the set of candidates is large, then there exist SNEs in which all of the candidates reject the DM's offers regardless of their availability.
} 
the first $t-1$ candidates who received offers are unavailable and that these candidates are the only ones whose value is greater than $v_{i}$. Hence, each available candidate who receives an offer infers that he is the most-valued among the available ones and accepts the DM's offer. From the DM's perspective, since there is no cost of delay in the model and available candidates always accept his offers, approaching candidates who are unlikely to be available is not costly. This makes it optimal for the DM to approach the candidates in the order of their values and allows him to avoid offending his most-preferred available candidate. This extreme example suggests that reference group considerations are likely to weaken the negative forces we identified in the paper.

\section{The benefit of randomising by delegation}

The DM in our model can strictly benefit from using the following two-stage procedure. In the first round, the DM narrows down the set of candidates $I$ to the top $z<n$ candidates on his preference list. In the second round, the DM commits to randomise uniformly by delegating the scheduling problem to an uninformed agent.

To demonstrate how the DM can benefit from such a procedure, consider the case of $k=1, c$ close to 1 , and assume that $p(v) v$ is constant. In any SNE of our baseline model, the DM's offers are accepted by available candidates in at most one round. To see this, observe that if $t$ and $t^{\prime}$ are the last two rounds in which offers are accepted by available candidates, then the optimality of the DM's strategy implies that the value of the candidate who receives an offer in round $t$ cannot be lower than the value of the candidate who receives an offer in round $t^{\prime}>t$. Therefore, accepting an offer in round $t^{\prime}$ cannot be a best response. The same argument implies that even if the DM were to commit to make offers only to the top $z>1$ candidates, the candidates would accept those offers in at most one round. Therefore, committing to make offers to the top $z$ candidates would have no effect on the DM's highest SNE payoff, which is $p(v) v$ in both cases. That is, narrowing down the set of candidates to the top ones without randomisation does not change the DM's payoff. How can randomisation help the DM to obtain a higher payoff?

If the DM could commit ex ante to make $z>1$ offers and randomise uniformly among the top $z<n$ candidates, then each candidate who receives an offer would believe that he was ranked first with a probability of more than $\frac{c-1}{c}$. Therefore, he would accept the DM's offer. As a result, the DM's payoff would be strictly greater than the maximal SNE payoff in the "no commitment" case and in the case of committing to make offers to the top $z$ candidates without the additional commitment to 
randomisation.

The fact that the decision is delegated to an uninformed party plays a key role in this setting as it allows the DM to hide information that may offend some candidates and make them reject his offers. This provides a rationale to hiring procedures that may seem inefficient at a glance. For instance, letting human resources have the ultimate say on whether a candidate should be hired or not (or letting a dean who is not particularly knowledgeable in a field decide on the the order of offers) can mitigate the adverse effect of social motives and increase the probability of hiring a suitable candidate.

\section{References}

[1] Alderfer, P. and McCord, C. (1970): "Personal and Situational Factors in the Recruitment Interview," Journal of Applied Psychology, 54(4), 377-385.

[2] Antler, Y. (2015): "Two-sided Matching with Endogenous Preferences," American Economic Journal: Microeconomics, 7(3), 241-258.

[3] Aronson, E. and Worchel, P. (1966): "Similarity Versus Liking as Determinants of Interpersonal Attractiveness," Psychonomic Science, 5(4), 157-158.

[4] Avery, C. and Levin, J. (2010): "Early Admissions at Selective Colleges," American Economic Review, 100(5), 2125-2156.

[5] Bretz, B., Gerhart, B., and Rynes, S. (1991): "The Importance of Recruitment in Job Choice: A Different Way of Looking," Personnel Psychology, 44(3), 487-521.

[6] Condon, J. and Crano, W. D. (1988): "Inferred Evaluation of the Relation between Attitude Similarity and Interpersonal Attraction," Journal of Personality and Social Psychology, 54(5), 789-797.

[7] Curtis, R. J. and Miller, K. (1986): "Believing Another Likes or Dislikes You: Behaviors Making the Beliefs Come True," Journal of Personality and Social Psychology, 51(2), 284-290.

[8] Gul, F. and Pesendorfer, W. (2016): "Interdependent Preference Models as a Theory of Intentions," Journal of Economic Theory, 165, 179-208.

[9] Jehiel, P. and Moldovanu, B. (1995): "Negative Externalities May Cause Delay in Negotiation," Econometrica, 63(6), 1321-1335. 
[10] Jehiel, P., Moldovanu, B., and Stacchetti, E. (1996): "How (Not) to Sell Nuclear Weapons," American Economic Review, 86(4), 814-829.

[11] Postlewaite, A. (1998): "The Social Basis of Interdependent Preferences," European Economic Review, 42, 779-800.

\section{Appendix}

\section{Proof of Claim 2}

Given the candidates' strategies, the DM is indifferent between both permutations. Therefore, it is left to check the candidates' strategies. Consider an arbitrary available candidate $i$. His interim belief about $v_{i}$ is

$$
f\left(v_{i} \mid s_{i}=1\right)=\frac{2(1-v)}{\int_{0.5}^{1} 2(1-v) d v}=8(1-v)
$$

It follows that

$$
\operatorname{Pr}\left(v_{i}>v_{j \neq i} \mid s_{i}=1\right)=\int_{0.5}^{1} 8(1-v) 2(v-0.5) d v=\frac{1}{3}
$$

Since $\sigma^{1}=0$ and the DM mixes uniformly between the two permutations, candidate $i$ does not receive any additional information. Since $c=2$, it follows that $\frac{1}{3}+\frac{2}{3}(1-c)<0$ and so rejecting the DM's offers is a best response in both rounds.

\section{Proof of Claim 3}

The proof is a special case of Claim 4. The nonexistence of an SNE in which offers are accepted with some probability in one of the rounds and always rejected in the other round (e.g., an SNE in which $\sigma^{1}>0$ and $\sigma^{2}=0$ ) follows from Case 1 of Claim 4. The fact that there exists no SNE in which the DM's offers are accepted in both rounds (i.e., an SNE in which $\sigma^{1}>0$ and $\sigma^{2}>0$ ) follows from Case 3 of Claim 4. 


\section{Claim 4 and asymmetric equilibria in the two-candidate example}

First, we shall demonstrate the existence of a Nash equilibrium in which the DM's strategy does not treat the candidates in a symmetric manner. Consider a profile of strategies in which the DM always makes the first offer to a specific candidate $i$ and both candidates always reject the DM's offers. Given the candidates' strategies, the DM's strategy is optimal. Since the DM's strategy is independent of the candidates' values, they do not learn anything from the fact that the DM approaches $i$ first. Candidate $i$ rejects the DM's offer independently of $s_{i}$ since, as we showed in (2), $\operatorname{Pr}\left(v_{i}>v_{j} \mid s_{i}=1\right)<\frac{1}{2}$. Therefore, the candidate $j$ who is approached second does not learn anything from the fact that $i$ rejected the first offer. This implies that, conditional on being available, it is a best response for $j$ to reject the DM's second offer. We can set the candidates' beliefs off the equilibrium path such that if $i$ (respectively, j) receives an offer in the second (respectively, first) round, then he believes that he is the least-valued candidate with probability 1 and rejects the DM's offer.

The above example demonstrates that when the symmetry assumption is relaxed, the candidates' beliefs need not have full support (the candidates' beliefs have full support if, in every round, each candidate believes that there is strictly positive probability that he will receive an offer). The example also shows that we are free to choose the candidates' beliefs off the equilibrium path as we wish. We would like to emphasise that we refrain from placing any restriction on the candidates' off-path beliefs since any such restriction would strengthen the solution concept and weaken the negative result of Claim 4.

Claim 4 In every Nash equilibrium, all of the DM's offers are rejected (regardless of the candidates' availability).

Proof. Since we relax the symmetry assumption for this claim, we must add a subscript to the description of each candidate's strategy. For every round $t \in\{1,2\}$ and every candidate $i$, denote by $\sigma_{i}^{t}$ the probability that candidate $i$ accepts an offer that is made in round $t$ when he is available. There are three cases that we need to address. In the first and second cases, there is no candidate $i$ for whom both $\sigma_{i}^{1}>0$ and $\sigma_{i}^{2}>0$. In the third case, there is at least one candidate who accepts the DM's offer with strictly positive probability in both rounds. 
Case 1: Nonexistence of a Nash equilibrium in which both candidates accept offers in round $t$ and reject them in round $t^{\prime} \neq t$.

Assume by negation that $\sigma_{i}^{t}>0, \sigma_{j}^{t}>0, \sigma_{i}^{t^{\prime}}=0$, and $\sigma_{j}^{t^{\prime}}=0$ are part of a Nash equilibrium. Consider round $t$. If $v_{i}\left(1-v_{i}\right) \sigma_{i}^{t}>v_{j}\left(1-v_{j}\right) \sigma_{j}^{t}$ (respectively, $v_{i}\left(1-v_{i}\right) \sigma_{i}^{t}<$ $\left.v_{j}\left(1-v_{j}\right) \sigma_{j}^{t}\right)$, then the DM's unique best response is to make an offer to candidate $i$ (respectively, $j$ ). Without loss of generality, assume that $\sigma_{i}^{t} \leq \sigma_{j}^{t}$. Observe that $v_{i}\left(1-v_{i}\right) \sigma_{i}^{t}>v_{j}\left(1-v_{j}\right) \sigma_{j}^{t}$ only if $v_{j}>v_{i}$. Hence, if candidate $i$ receives an offer in round $t$, then he must infer that $v_{i}<v_{j}$, and, therefore, reject this offer. This is in contradiction to the assumption that $\sigma_{i}^{t}>0$ is part of a Nash equilibrium.

In the next two cases, the candidates' beliefs need not have full support. Before we proceed to the proofs of Cases 2 and 3, it will be useful to show that if the candidates' beliefs do not have full support, then it must be that $\sigma_{i}^{1}=\sigma_{j}^{1}=\sigma_{i}^{2}=\sigma_{j}^{2}=0$.

If the candidates' beliefs do not have full support, then there exists a permutation $\pi^{\star}$ such that $\int_{0.5}^{1} \int_{0.5}^{1} 4 \alpha\left(\pi^{\star} \mid v_{i}, v_{j}\right) d v_{i} d v_{j}=1$. Consider the candidate $i$ who receives an offer first according to $\pi^{\star}$. Since the DM approaches candidate $i$ first with probability 1 , the latter does not receive any additional information from the fact that he receives an offer in round 1. Formally, conditional on both $s_{i}=1$ and receiving an offer in round 1 , candidate $i$ believes that $v_{i}>v_{j}$ with a probability of

$$
\frac{\int_{0.5}^{1} \int_{0.5}^{v_{i}} 4 \alpha\left(\pi^{\star} \mid v_{i}, v_{j}\right)\left(1-v_{i}\right) d v_{i} d v_{j}}{\int_{0.5}^{1} \int_{0.5}^{1} 4 \alpha\left(\pi^{\star} \mid v_{i}, v_{j}\right)\left(1-v_{i}\right) d v_{i} d v_{j}}=\frac{\int_{0.5}^{1} \int_{0.5}^{v_{i}}\left(1-v_{i}\right) d v_{i} d v_{j}}{\int_{0.5}^{1} \int_{0.5}^{1}\left(1-v_{i}\right) d v_{i} d v_{j}}=\frac{1}{3}
$$

Since $c=2$, it follows that $\frac{1}{3}+\frac{2}{3}(1-c)<0$, such that rejecting the DM's first offer is the unique best response for candidate $i$. It follows that $\sigma_{i}^{1}=0$. Hence, candidate $j \neq i$ does not learn any information from the fact that $i$ rejects the first offer. Using a similar argument, it is possible to show that, conditional on both receiving an offer second and $s_{j}=1$, candidate $j$ believes that $v_{j}>v_{i}$ with probability $\frac{1}{3}$. Thus, rejecting the DM's second offer is the unique best response for candidate $j$. Observe that since $\sigma_{i}^{1}=\sigma_{j}^{2}=0$, for (almost) every realisation, the DM's payoff is 0 in this equilibrium. It follows that $\sigma_{i}^{2}=\sigma_{j}^{1}=0$ (otherwise, the DM could make a strictly positive expected payoff by deviating to a different permutation).

Case 2: Nonexistence of a Nash equilibrium in which $\sigma_{i}^{t}>0, \sigma_{j}^{t}=0$, and $\sigma_{i}^{t^{\prime} \neq t}=0$. 
Assume by negation that $\sigma_{i}^{t}>0, \sigma_{j}^{t}=0$, and $\sigma_{i}^{t^{\prime} \neq t}=0$ are part of a Nash equilibrium. The DM's unique ${ }^{5}$ best response is to approach candidate $i$ (respectively, $j$ ) in round $t$ (respectively, $t^{\prime} \neq t$ ) since any other strategy guarantees him a payoff of 0 . Thus, the candidates' beliefs do not have full support. The rest of the proof follows from the above argument.

Case 3: Nonexistence of a Nash equilibrium in which $\sigma_{i}^{1}>0$ and $\sigma_{i}^{2}>0$.

Assume by negation that $\sigma_{i}^{1}>0$ and $\sigma_{i}^{2}>0$ are part of a Nash equilibrium. Either the candidates' beliefs have full support or not. Since the latter case is already proven, we focus on the former one and assume that the candidates' beliefs have full support. Let $\pi^{\star}$ be a permutation under which $i$ receives an offer in round 1 . The LHS of the next inequality, which represents $i$ 's belief that he is the most-valued candidate conditional on being available and receiving an offer in round 1 , is well defined since $i$ 's beliefs have full support. It is greater than $\frac{1}{2}$ since $\sigma_{i}^{1}>0$ is part of a Nash equilibrium and $c=2$.

$$
\frac{\int_{0.5}^{1} \int_{0.5}^{v_{i}} \alpha\left(\pi^{\star} \mid v_{i}, v_{j}\right)\left(1-v_{i}\right) d v_{i} d v_{j}}{\int_{0.5}^{1} \int_{0.5}^{1} \alpha\left(\pi^{\star} \mid v_{i}, v_{j}\right)\left(1-v_{i}\right) d v_{i} d v_{j}} \geq \frac{1}{2}
$$

The LHS of the next inequality, which represents candidate $i$ 's belief that he is the mostvalued candidate, conditional on receiving an offer in round 2 and being available, is also well defined by our "full support" assumption. It is greater than $\frac{1}{2}$ since $\sigma_{i}^{2}>0$ is part of a Nash equilibrium and $c=2$.

$$
\frac{\int_{0.5}^{1} \int_{0.5}^{v_{i}}\left(1-\alpha\left(\pi^{\star} \mid v_{i}, v_{j}\right)\right)\left(1-v_{i}\right)\left(1-\sigma_{j}^{1}\left(1-v_{j}\right)\right) d v_{i} d v_{j}}{\int_{0.5}^{1} \int_{0.5}^{1}\left(1-\alpha\left(\pi^{\star} \mid v_{i}, v_{j}\right)\right)\left(1-v_{i}\right)\left(1-\sigma_{j}^{1}\left(1-v_{j}\right)\right) d v_{i} d v_{j}} \geq \frac{1}{2}
$$

We can transform inequality (4) to inequality (6) and inequality (5) to inequality (7).

$$
\begin{gathered}
\int_{0.5}^{1} \int_{0.5}^{v_{i}} \alpha\left(\pi^{\star} \mid v_{i}, v_{j}\right)\left(1-v_{i}\right) d v_{i} d v_{j} \geq \int_{0.5}^{1} \int_{v_{i}}^{1} \alpha\left(\pi^{\star} \mid v_{i}, v_{j}\right)\left(1-v_{i}\right) d v_{i} d v_{j} \\
\int_{0.5}^{1} \int_{0.5}^{v_{i}}\left(1-\alpha\left(\pi^{\star} \mid v_{i}, v_{j}\right)\right)\left(1-v_{i}\right)\left(1-\sigma_{j}^{1}\left(1-v_{j}\right)\right) d v_{i} d v_{j} \geq \\
\quad \int_{0.5}^{1} \int_{v_{i}}^{1}\left(1-\alpha\left(\pi^{\star} \mid v_{i}, v_{j}\right)\right)\left(1-v_{i}\right)\left(1-\sigma_{j}^{1}\left(1-v_{j}\right)\right) d v_{i} d v_{j}
\end{gathered}
$$

\footnotetext{
${ }^{5}$ Except for the case of $v_{i}=v_{j}=1$ in which the DM is indifferent between the two permutations.
} 
We can sum over the LHS and RHS of (6) and (7) and rearrange to obtain

$$
\begin{array}{r}
\int_{0.5}^{1} \int_{0.5}^{v_{i}}\left(1-v_{i}\right) d v_{i} d v_{j}-\int_{0.5}^{1} \int_{v_{i}}^{1}\left(1-v_{i}\right) d v_{i} d v_{j} \geq \\
\int_{0.5}^{1} \int_{0.5}^{v_{i}}\left(1-\alpha\left(\pi^{\star} \mid v_{i}, v_{j}\right)\right) \sigma_{j}^{1}\left(1-v_{i}\right)\left(1-v_{j}\right) d v_{i} d v_{j}- \\
\int_{0.5}^{1} \int_{v_{i}}^{1}\left(1-\alpha\left(\pi^{\star} \mid v_{i}, v_{j}\right)\right) \sigma_{j}^{1}\left(1-v_{i}\right)\left(1-v_{j}\right) d v_{i} d v_{j}
\end{array}
$$

The LHS of (8) is independent of $\alpha$ and equals $\frac{-1}{48}$. The RHS of (8) is minimised when $\sigma_{j}^{1}=1$ and $\alpha\left(v^{\star} \mid v_{i}, v_{j}\right)=0$ (respectively, $\alpha\left(v^{\star} \mid v_{i}, v_{j}\right)=1$ ) if $v_{j}>v_{i}$ (respectively, $v_{i}>$

$\left.v_{j}\right)$. In that case, the RHS of (8) equals $-\int_{0.5}^{1} \int_{v_{i}}^{1}\left(1-v_{i}\right)\left(1-v_{j}\right) d v_{i} d v_{j}=\frac{-1}{128}$. Hence, conditions (4) and (5) cannot both hold. This is in contradiction to the assumption that there exists a Nash equilibrium in which both $\sigma_{i}^{1}>0$ and $\sigma_{i}^{2}>0$.

\section{Proof of Proposition 1}

Let $\sigma^{1}=\ldots=\sigma^{n}=0$. Clearly, each strategy is a best response for the DM. Since $p(v)$ is weakly decreasing in $v$, each available candidate believes that he is one of the DM's $k$-most-valued candidates with a probability of at most $\frac{k}{n}$. Since $\sigma^{1}=\ldots=\sigma^{n}=0$ and the DM mixes uniformly among permutations, the candidates do not receive any additional information. Since, by assumption, $\frac{k}{n} \leq \frac{c-1}{c}$, it follows that $\sigma^{1}=\ldots=\sigma^{n}=0$ are best responses.

\section{Proof of Proposition 2}

Before we proceed with the proof, we need some additional notation. We denote a profile of candidates' strategies $\left(\sigma^{1}, \ldots, \sigma^{n}\right)$ by $\sigma$. For each realisation $\left(v_{1}, \ldots, v_{n}\right)$, permutation $\pi$, and profile of candidates' strategies $\sigma$, we use $V_{t}\left(\pi, \sigma, v_{1}, \ldots, v_{n}\right)$ to denote the continuation value of a DM who schedules offers according to the permutation $\pi$ (from round 1 ) and all of his offers in rounds $1, \ldots, t-1$ were rejected. Define $V_{t}\left(\alpha, \sigma, v_{1}, \ldots, v_{n}\right):=\min _{\pi \mid \alpha\left(\pi \mid v_{1}, \ldots, v_{n}\right)>0} V_{t}\left(\pi, \sigma, v_{1}, \ldots, v_{n}\right)$. Let $T(\sigma):=\left\{t \in N \mid \sigma^{t}>0\right\}$ denote the set of rounds in which the DM's offers might be accepted given the profile of candidates' strategies $\sigma$.

The candidate who receives an offer in round $t$ is said to be active if he is available and the offers in rounds $1, \ldots, t-1$ were rejected. We denote the probability that the candidate who receives an offer in round $t$ is active given the profile of strategies $(\alpha, \sigma)$ 
by $\lambda_{t}(\alpha, \sigma)$. We can write $\lambda_{t}(\alpha, \sigma)$ as

$$
\begin{array}{r}
\int_{v_{l}}^{v_{h}} \ldots \int_{v_{l}}^{v_{h}} \sum_{\pi} \alpha\left(\pi \mid v_{1}, \ldots, v_{n}\right)\left(1-\sigma^{1} p\left(v_{\pi_{1}\left(v_{1}, \ldots, v_{n}\right)}\right)\right) \ldots \\
\left(1-\sigma^{t-1} p\left(v_{\pi_{t-1}\left(v_{1}, \ldots, v_{n}\right)}\right)\right) p\left(v_{\pi_{t}\left(v_{1}, \ldots, v_{n}\right)}\right) f\left(v_{1}\right) \ldots f\left(v_{n}\right) d v_{1} \ldots d v_{n}
\end{array}
$$

Denote the probability that the candidate who receives an offer in round $t$ is active and the candidate who receives an offer in round $r$ is one of the $k$-most-valued candidates by $\lambda_{t r}(\alpha, \sigma)$. Observe that $t$ and $r$ need not be the same round. We can write $\lambda_{t r}(\alpha, \sigma)$ as

$$
\begin{array}{r}
\int_{v_{l}}^{v_{h}} \ldots \int_{v_{l}}^{v_{h}} \sum_{\pi} \alpha\left(\pi \mid v_{1}, \ldots, v_{n}\right) \mathbb{1}_{r}\left(\pi, v_{1}, \ldots, v_{n}\right)\left(1-\sigma^{1} p\left(v_{\pi_{1}\left(v_{1}, \ldots, v_{n}\right)}\right)\right) \ldots \\
\quad\left(1-\sigma^{t-1} p\left(v_{\pi_{t-1}\left(v_{1}, \ldots, v_{n}\right)}\right)\right) p\left(v_{\pi_{t}\left(v_{1}, \ldots, v_{n}\right)}\right) f\left(v_{1}\right) \ldots f\left(v_{n}\right) d v_{1} \ldots d v_{n}
\end{array}
$$

where $\mathbb{1}_{r}\left(\pi, v_{1}, \ldots, v_{n}\right) \in\{0,1\}$ is an indicator that equals 1 if and only if the candidate who receives an offer in round $r \in N$ according to $\pi$ is one of the $k$-most-valued candidates, that is, if $x_{\pi_{r}\left(v_{1}, \ldots, v_{n}\right)}<k$. Observe that $\frac{\lambda_{t t}(\alpha, \sigma)}{\lambda_{t}(\alpha, \sigma)}$ is the probability that a candidate who is active in round $t$ assigns to the event that he is one of the $k$-mostvalued candidates given the profile $(\alpha, \sigma)$.

Let $\left(\hat{\alpha}_{n}, \hat{\sigma}_{n}\right)_{n=1}^{\infty}$ be a sequence of SNEs such that $\left(\hat{\alpha}_{n}, \hat{\sigma}_{n}\right)$ is played in the game with $n$ candidates. Our objective in the following four lemmata is to show that there exists a number $T>0$ such that $\left|T\left(\hat{\sigma}_{n}\right)\right|<T$ for every $n \in \mathbb{N}$ (that is, to show that the number of rounds in which the DM's offers might be accepted is bounded from above). Let us assume by negation that this is not the case. For each $m \in \mathbb{N}$, define $n(m)$ to be the lowest $n$ for which $\left|T\left(\hat{\sigma}_{n}\right)\right| \geq m$. Observe that $n(m) \geq m$. For every $m \in \mathbb{N}$, denote $\left(\tilde{\alpha}_{m}, \tilde{\sigma}_{m}\right):=\left(\hat{\alpha}_{n(m)}, \hat{\sigma}_{n(m)}\right)$. That is, $\left(\tilde{\alpha}_{m}, \tilde{\sigma}_{m}\right)_{m=1}^{\infty}$ is a sequence of SNEs such that $\left(\tilde{\alpha}_{m}, \tilde{\sigma}_{m}\right)$ is played in the game with $n(m)$ candidates and $\left|T\left(\tilde{\sigma}_{m}\right)\right| \geq m$.

For each $m \in \mathbb{N}$ and $z=1, \ldots, m$, define $T_{z m}$ to be an arbitrary subset of $T\left(\tilde{\sigma}_{m}\right)$ that satisfies

(a) $\left|T_{z m}\right|=z$.

(b) If $t, t^{\prime \prime} \in T_{z m}, t^{\prime} \notin T_{z m}$, and $t>t^{\prime}>t^{\prime \prime}$, then $t^{\prime} \notin T\left(\tilde{\sigma}_{m}\right)$.

Lemma 1 will show that there is a number of rounds $z$ such that for every $m \geq z$, every set of rounds $T_{z m}$ includes at least one round $t$ in which offers are accepted with a probability of more than $\epsilon_{z}$ (i.e., $\sigma^{t} \geq \epsilon_{z}$ ), where $\epsilon_{z}>0$ is independent of $m$.

Lemma 1 There exist two numbers, $z \in \mathbb{N}$ and $\epsilon_{z} \in(0,1)$, such that for each $m \geq z$ 
and each $T_{z m}$, there exists a round $t \in T_{z m}$ such that $\tilde{\sigma}_{m}^{t} \geq \epsilon_{z}$.

Proof. Consider $z>2 k, m \geq z, T_{z m} \subseteq T\left(\tilde{\sigma}_{m}\right)$, and use $\lambda_{z m}^{\star}$ to denote the median value of $\left\{\lambda_{t}\left(\tilde{\alpha}_{m}, \tilde{\sigma}_{m}\right) \mid t \in T_{z m}\right\}$. Define two disjoint sets of $\left\lfloor\frac{z}{2}\right\rfloor$ rounds, $T_{z m}^{\star} \subset T_{z m}$ and $T_{z m}^{\star \star} \subset T_{z m}$, such that for each $t \in T_{z m}^{\star}, \lambda_{t}\left(\tilde{\alpha}_{m}, \tilde{\sigma}_{m}\right) \leq \lambda_{z m}^{\star}$, and for each $t \in T_{z m}^{\star \star}$, $\lambda_{t}\left(\tilde{\alpha}_{m}, \tilde{\sigma}_{m}\right) \geq \lambda_{z m}^{\star}$.

Consider $r \in T_{z m}$. Let us look at (10) for $\lambda_{t r}\left(\tilde{\alpha}_{m}, \tilde{\sigma}_{m}\right)$. The weight of each realisation $\left(v_{1}, \ldots, v_{n}\right)$ and permutation $\pi$ such that $\tilde{\alpha}_{m}\left(\pi \mid v_{1}, \ldots, v_{n}\right) \mathbb{1}_{r}\left(\pi, v_{1}, \ldots, v_{n}\right)>0$ is the probability that $t-1$ offers were rejected and that $\pi_{t}\left(v_{1}, \ldots, v_{n}\right)$ is available. It is given in (11).

$$
\begin{array}{r}
w_{t}\left(\tilde{\sigma}_{m}, \pi, v_{1}, \ldots, v_{n(m)}\right)=\left(1-\tilde{\sigma}_{m}^{1} p\left(v_{\pi_{1}\left(v_{1}, \ldots, v_{n(m)}\right)}\right)\right) \ldots \\
\left(1-\tilde{\sigma}_{m}^{t-1} p\left(v_{\pi_{t-1}\left(v_{1}, \ldots, v_{n(m)}\right)}\right)\right) p\left(v_{\pi_{t}\left(v_{1}, \ldots, v_{n(m)}\right)}\right)
\end{array}
$$

Since $\mathbb{1}_{r}\left(\pi, v_{1}, \ldots, v_{n}\right)=1$, there are at least $\left\lfloor\frac{z}{2}\right\rfloor-k$ candidates who receive offers in rounds $t \in T_{z m}^{\star}$ for whom $v_{\pi_{t}\left(v_{1}, \ldots, v_{n}\right)} \leq v_{\pi_{r}\left(v_{1}, \ldots, v_{n}\right)}$, and since $p(v)$ is decreasing in $v$, $p\left(v_{\pi_{t}\left(v_{1}, \ldots, v_{n}\right)}\right) \geq p\left(v_{\pi_{r}\left(v_{1}, \ldots, v_{n}\right)}\right)$. Denote $\epsilon:=\max \left\{\tilde{\sigma}_{m}^{t} \mid t \in T_{z m}\right\}$. Since $p(v) \leq 1$, and $\tilde{\sigma}_{m}^{i} \leq \epsilon$ for each $i \in T_{z m}$,

$$
w_{t}\left(\tilde{\sigma}_{m}, \pi, v_{1}, \ldots, v_{n(m)}\right) \geq w_{r}\left(\tilde{\sigma}_{m}, \pi, v_{1}, \ldots, v_{n(m)}\right)(1-\epsilon)^{z}
$$

for each round $t$ that is one of the $\left\lfloor\frac{z}{2}\right\rfloor-k$ rounds in $T_{z m}^{\star}$ in which $p\left(v_{\pi_{t}\left(v_{1}, \ldots, v_{n}\right)}\right) \geq$ $p\left(v_{\pi_{r}\left(v_{1}, \ldots, v_{n}\right)}\right)$. Observe that in the last transition we used property (b) and the fact that each round $t \notin T\left(\tilde{\sigma}_{m}\right)$ can be ignored since $\tilde{\sigma}_{m}^{t}=0$. It follows that $\sum_{t \in T_{z m}^{\star}} \lambda_{t r}\left(\tilde{\alpha}_{m}, \tilde{\sigma}_{m}\right) \geq(1-\epsilon)^{z}\left(\left\lfloor\frac{z}{2}\right\rfloor-k\right) \lambda_{r r}\left(\tilde{\alpha}_{m}, \tilde{\sigma}_{m}\right)$ for each $r \in T_{z m}$.

Since $\frac{\lambda_{t r}\left(\tilde{\alpha}_{m}, \tilde{\sigma}_{m}\right)}{\lambda_{t}\left(\tilde{\alpha}_{m}, \tilde{\sigma}_{m}\right)}$ is the probability that a candidate who is active in the $t$-th round assigns to the event that the candidate who receives an offer in the $r$-th round is one of the $k$-most-valued candidates, it follows that $\sum_{r \in T_{z m}} \frac{\lambda_{t r}\left(\tilde{\alpha}_{m}, \tilde{\sigma}_{m}\right)}{\lambda_{t}\left(\tilde{\alpha}_{m}, \tilde{\sigma}_{m}\right)} \leq k$. Therefore,

$$
\begin{array}{r}
k z \geq \sum_{t \in T_{z m}} \sum_{r \in T_{z m}} \frac{\lambda_{t r}\left(\tilde{\alpha}_{m}, \tilde{\sigma}_{m}\right)}{\lambda_{t}\left(\tilde{\alpha}_{m}, \tilde{\sigma}_{m}\right)}>\sum_{t \in T_{z m}^{\star}} \sum_{r \in T_{z m}^{\star \star}} \frac{\lambda_{t r}\left(\tilde{\alpha}_{m}, \tilde{\sigma}_{m}\right)}{\lambda_{t}\left(\tilde{\alpha}_{m}, \tilde{\sigma}_{m}\right)} \geq \\
\sum_{t \in T_{z m}^{\star}} \sum_{r \in T_{z m}^{\star \star}} \frac{\lambda_{t r}\left(\tilde{\alpha}_{m}, \tilde{\sigma}_{m}\right)}{\lambda_{z m}^{\star}} \geq(1-\epsilon)^{z} \sum_{t \in T_{z m}^{\star \star}} \frac{\left(\left\lfloor\frac{z}{2}\right\rfloor-k\right) \lambda_{t t}\left(\tilde{\alpha}_{m}, \tilde{\sigma}_{m}\right)}{\lambda_{z m}^{\star}} \geq \\
(1-\epsilon)^{z}\left(\left\lfloor\frac{z}{2}\right\rfloor-k\right)\left\lfloor\frac{z}{2}\right\rfloor \frac{c-1}{c}
\end{array}
$$


It follows that for a sufficiently large $z$ and $m \geq z$, it must be that $\epsilon$ is greater than some $\epsilon_{z}>0$ that is independent of $m$.

Lemma 1 establishes that for large values of $m$, at least $\left\lfloor\frac{m}{z}\right\rfloor$ of the DM's offers are accepted (by available candidates) with a probability of more than $\epsilon_{z}>0$. In Lemmata 2 and 3, we will consider large values of $m$. Fix arbitrary $\delta \in(0,1)$ and $\bar{\epsilon} \in\left(0, \frac{v_{h}-v_{l}}{2}\right)$. For every $m \in \mathbb{N}$, let $\left(\tilde{v}_{1}, \ldots, \tilde{v}_{n(m)}\right)$ be an arbitrary realisation of candidates' values such that $\left|\left\{i \mid v_{i} \in\left(v_{h}-2 \bar{\epsilon}, v_{h}-\bar{\epsilon}\right)\right\}\right| \geq \delta m$. Observe that if $\delta<F\left(v_{h}-\bar{\epsilon}\right)-F\left(v_{h}-2 \bar{\epsilon}\right)$, then the probability of such a realisation approaches 1 as $m$ goes to infinity.

Lemma 2 Fix $t \in \mathbb{N}$. There exists a number $m^{t} \in \mathbb{N}$ such that for each $m \geq m^{t}$, $V_{j}\left(\tilde{\alpha}_{m}, \tilde{\sigma}_{m}, \tilde{v}_{1}, \ldots, \tilde{v}_{n(m)}\right)>v_{h}-3 \bar{\epsilon}$ for each $j \in\left\{t^{\prime} \in T\left(\tilde{\sigma}_{m}\right):\left|\left\{\bar{t} \in T\left(\tilde{\sigma}_{m}\right): \bar{t} \leq t^{\prime}\right\}\right| \leq t\right\}$ (i.e., for each of the first $t$ rounds of $T(\tilde{\sigma})$ ).

Proof. Denote the following strategy by $\alpha^{\prime}$ : in each round $t \in T\left(\tilde{\sigma}_{m}\right)$ make an offer to the lowest-valued candidate among those with value $v>v_{h}-2 \bar{\epsilon}$ who did not receive an offer previously. If such a candidate does not exist, make an offer to the highest-valued candidate who did not receive an offer previously. In each round $t \notin T\left(\tilde{\sigma}_{m}\right)$ make an offer to an arbitrary candidate whose value is $v \leq v_{h}-2 \bar{\epsilon}$. If such a candidate does not exist, make an offer to the highest-valued candidate who did not receive an offer previously.

For $j=1, \ldots,\left|T\left(\tilde{\sigma}_{m}\right)\right|$, denote the following strategy by $\beta_{j}$. First, the DM chooses an arbitrary permutation $\pi$ such that $\tilde{\alpha}_{m}\left(\pi \mid \tilde{v}_{1}, \ldots, \tilde{v}_{n(m)}\right)>0$. Up to the $j$-th round of $T\left(\tilde{\sigma}_{m}\right)$, the DM makes the offers in each round $t \in T\left(\tilde{\sigma}_{m}\right)$ according to $\pi$. In the remaining rounds (i.e., $N / T\left(\tilde{\sigma}_{m}\right)$ ), the DM makes the offers according to the rule that defines $\alpha^{\prime}$. Let us denote the $j$-th round of $T\left(\tilde{\sigma}_{m}\right)$ by $j^{\star}$. By the optimality of $\tilde{\alpha}_{m}$, it must be that $V_{j^{\star}}\left(\beta_{j}, \tilde{\sigma}_{m}, \tilde{v}_{1}, \ldots, \tilde{v}_{n(m)}\right) \leq V_{j^{\star}}\left(\pi, \tilde{\sigma}_{m}, \tilde{v}_{1}, \ldots, \tilde{v}_{n(m)}\right)$. Moreover, if $t<j$ and $t^{\star}$ is the $t$-th round of $T\left(\tilde{\sigma}_{m}\right)$, then $V_{t^{\star}}\left(\beta_{t}, \tilde{\sigma}_{m}, \tilde{v}_{1}, \ldots, \tilde{v}_{n(m)}\right) \leq V_{t^{\star}}\left(\pi, \tilde{\sigma}_{m}, \tilde{v}_{1}, \ldots, \tilde{v}_{n(m)}\right)$.

By construction, according to $\beta_{j}$, in the rounds $\left\{t^{\prime} \in T\left(\tilde{\sigma}_{m}\right) \mid t^{\prime} \geq j^{\star}\right\}$, the DM makes at least $\lfloor\delta m\rfloor-j+1$ offers to candidates with a value $v \in\left(v_{h}-2 \bar{\epsilon}, v_{h}-\bar{\epsilon}\right)$ when he faces a realisation $\left(\tilde{v}_{1}, \ldots, \tilde{v}_{n(m)}\right)$. By Lemma 1 , there is a number $z \in \mathbb{N}$ such that at least $\left\lfloor\frac{\lfloor\delta m\rfloor-j+1}{z}\right\rfloor$ of these offers are accepted with a probability of more than $\epsilon_{z} p\left(v_{h}-\bar{\epsilon}\right)>0$. If we chose a sufficiently large $m$, then $V_{j^{\star}}\left(\beta_{j}, \tilde{\sigma}_{m}, \tilde{v}_{1}, \ldots, \tilde{v}_{n(m)}\right)>v_{h}-3 \bar{\epsilon}$. If $t<j$ and $t^{\star}$ is the $t$-th round of $T\left(\tilde{\sigma}_{m}\right)$, then $V_{t^{\star}}\left(\beta_{t}, \tilde{\sigma}_{m}, \tilde{v}_{1}, \ldots, \tilde{v}_{n(m)}\right)>v_{h}-3 \bar{\epsilon}$. Since we made no restriction about $\pi$, it follows that for a sufficiently large $m$, for each of the first $t$ rounds of $T(\tilde{\sigma}), V_{t}\left(\tilde{\alpha}_{m}, \tilde{\sigma}_{m}, \tilde{v}_{1}, \ldots, \tilde{v}_{n(m)}\right)>v_{h}-3 \bar{\epsilon}$.

In Lemma 2 we considered the first $t$ rounds of $T\left(\tilde{\sigma}_{m}\right)$. We showed that for large values of $m$, the DM's continuation value in these rounds is arbitrarily close to $v_{h}$ when 
he faces a realisation $\left(\tilde{v}_{1}, \ldots, \tilde{v}_{n(m)}\right)$. We now exploit this lemma to show that when $m$ is large, in the first $t$ rounds of $T(\tilde{\sigma})$ the DM approaches candidates whose value is arbitrarily close to $v_{h}$.

Lemma 3 Fix $t \in \mathbb{N}, \epsilon>0$. There exists a number $m(t, \epsilon)$ such that for each $m \geq$ $m(t, \epsilon)$, if the DM faces a realisation $\left(\tilde{v}_{1}, \ldots, \tilde{v}_{m(n)}\right)$ and makes an offer to a candidate with a value $v<v_{h}-4 \bar{\epsilon}$ in round $j \in\left\{t^{\prime} \in T\left(\tilde{\sigma}_{m}\right):\left|\left\{\bar{t} \in T\left(\tilde{\sigma}_{m}\right): \bar{t} \leq t^{\prime}\right\}\right| \leq t\right\}$, then $\tilde{\sigma}_{m}^{j}<\epsilon$.

Proof. Consider candidate $i$ who (according to $\pi_{m}$ such that $\tilde{\alpha}_{m}\left(\pi_{m} \mid \tilde{v}_{1}, \ldots, \tilde{v}_{n(m)}\right)>0$ ) is scheduled to receive an offer in round $j$, which is one of the first $t$ rounds of $T\left(\tilde{\sigma}_{m}\right)$, and suppose that $v_{i}<v_{h}-4 \bar{\epsilon}$. Since $\tilde{\alpha}_{m}\left(\pi_{m} \mid \tilde{v}_{1}, \ldots, \tilde{v}_{n(m)}\right)>0$, then, by definition,

$$
V_{j}\left(\pi_{m}, \tilde{\sigma}_{m}, \tilde{v}_{1}, \ldots, \tilde{v}_{n(m)}\right)=\tilde{\sigma}^{j} p\left(\tilde{v}_{i}\right) \tilde{v}_{i}+\left(1-\tilde{\sigma}^{j} p\left(\tilde{v}_{i}\right)\right) V_{j+1}\left(\pi_{m}, \tilde{\sigma}_{m}, \tilde{v}_{1}, \ldots, \tilde{v}_{n(m)}\right)
$$

By Lemma 2, there exists a number $m^{j+1}$ such that if $m>m^{j+1}$, then $v_{h}-3 \bar{\epsilon}<$ $V_{j}\left(\tilde{\alpha}_{m}, \tilde{\sigma}_{m}, \tilde{v}_{1}, \ldots, \tilde{v}_{n(m)}\right)$ and $v_{h}-3 \bar{\epsilon}<V_{j+1}\left(\tilde{\alpha}_{m}, \tilde{\sigma}_{m}, \tilde{v}_{1}, \ldots, \tilde{v}_{n(m)}\right)$. Consider $m>m^{j+1}$.

Suppose that there exists a round $r$ such that $\tilde{\sigma}_{m}^{r}=0$. It must be that in round $r$ the DM makes an offer to a candidate $i^{\prime}$ with a value $v_{i^{\prime}} \leq v_{i}$. Otherwise, the DM could do better by switching between $i$ and $i^{\prime}$, which is in contradiction to the optimality of $\pi_{m}$ and $\tilde{\alpha}_{m}$.

It follows that by round $j$ (which is one of the first $t$ rounds of $T\left(\tilde{\sigma_{m}}\right)$ ) the DM makes at most $t-1$ offers to candidates with a value $v \in\left(v_{h}-2 \bar{\epsilon}, v_{h}-\bar{\epsilon}\right)$. There are at least $\lfloor\delta m\rfloor-t+1$ candidates with a value $v \in\left(v_{h}-2 \bar{\epsilon}, v_{h}-\bar{\epsilon}\right)$ who do not receive an offer by round $j$. Denote the set of rounds in which they are scheduled to receive offers according to $\pi_{m}$ by $Q$. Let $\tilde{\sigma}_{m}^{\text {min }}:=\min \left\{\tilde{\sigma}_{m}^{t}: t \in Q\right\}$.

Suppose that the game has reached round $j$ (that is, the DM's offers in rounds $1, \ldots, j-1$ were rejected). The probability that the last (according to $\pi_{m}$ ) of the candidates with a value $v \in\left(v_{h}-2 \bar{\epsilon}, v_{h}-\bar{\epsilon}\right)$ will receive an offer must be lower than $\left(1-\tilde{\sigma}_{m}^{\text {min }} p\left(v_{h}-\bar{\epsilon}\right)\right)^{\lfloor\delta m\rfloor-t}$ as this happens only if $\lfloor\delta m\rfloor-t$ such candidates reject the DM's offers. For each $\epsilon^{\prime}>0$, there exists $m^{\epsilon^{\prime}} \in \mathbb{N}$ such that for each $m>m^{\epsilon^{\prime}}, \tilde{\sigma}_{m}^{\text {min }}<\epsilon^{\prime}$ or $\left(1-\tilde{\sigma}_{m}^{\min } p\left(v_{h}-\bar{\epsilon}\right)\right)^{\lfloor\delta m\rfloor-t}<\epsilon^{\prime}$. This implies that for a sufficiently large $m$, the DM could switch between $i$ (who receives an offer in round $j$ and has a value of $v_{i}<v_{h}-4 \bar{\epsilon}$ ) and a candidate with a value $v_{i^{\prime}}>v_{h}-2 \bar{\epsilon}$, while making an arbitrarily small change to $V_{j+1}\left(\pi_{m}, \tilde{\sigma}_{m}, \tilde{v}_{1}, \ldots, \tilde{v}_{n(m)}\right)$. Since $p\left(v_{i^{\prime}}\right) \leq p\left(v_{i}\right)$ and $V_{j+1}\left(\tilde{\alpha}_{m}, \tilde{\sigma}_{m}, \tilde{v}_{1}, \ldots, \tilde{v}_{n(m)}\right)>$ $v_{h}-3 \bar{\epsilon}$, if $\tilde{\sigma}^{j} \geq \epsilon$, we get a contradiction to the optimality of $\pi_{m}$ and $\tilde{\alpha}_{m}$. 
In Lemma 4 we show that the number of rounds in which the DM's offers might be accepted in an SNE is bounded from above.

Lemma 4 There exists a number $T>0$ such that for every $n \in \mathbb{N},\left|T\left(\hat{\sigma}_{n}\right)\right|<T$.

Proof. Assume to the contrary that the claim is false. Then, the sequence $\left(\tilde{\alpha}_{m}, \tilde{\sigma}_{m}\right)_{m=1}^{\infty}$ can be defined as before. Fix an arbitrarily small $\bar{\epsilon}>0$ and let $\delta(\bar{\epsilon}):=\frac{F\left(v_{h}-\bar{\epsilon}\right)-F\left(v_{h}-2 \bar{\epsilon}\right)}{2}$. By Lemma 3, for every $t \in \mathbb{N}$ and $\epsilon>0$, there exists a number $m(t, \epsilon)$ such that if $m>m(t, \epsilon)$ and $\left|\left\{i \mid v_{i} \in\left(v_{h}-2 \bar{\epsilon}, v_{h}-\bar{\epsilon}\right)\right\}\right| \geq \delta(\bar{\epsilon}) m$, then in each round $j$ such that $j \in\left\{t^{\prime} \in T\left(\tilde{\sigma}_{m}\right):\left|\left\{\bar{t} \in T\left(\tilde{\sigma}_{m}\right): \bar{t} \leq t^{\prime}\right\}\right| \leq t\right\}$, the DM makes an offer to a candidate with a value $v>v_{h}-4 \bar{\epsilon}$ or $\tilde{\sigma}_{m}^{j}<\epsilon$.

Let $B_{t}=\left\{t^{\prime} \in T\left(\tilde{\sigma}_{m}\right):\left|\left\{\bar{t} \in T\left(\tilde{\sigma}_{m}\right): \bar{t} \leq t^{\prime}\right\}\right| \leq t\right\}$ and consider $\lambda_{j r}\left(\tilde{\alpha}_{m}, \tilde{\sigma}_{m}\right)$, where $j \in B_{t}$ and $r \in N$. Observe that the probability that the values' realisation is such that $\left|\left\{i \mid v_{i} \in\left(v_{h}-2 \bar{\epsilon}, v_{h}-\bar{\epsilon}\right)\right\}\right| \geq \delta(\bar{\epsilon}) m$ approaches 1 as $m$ goes to infinity. Let us consider two such realisations, $\left(\tilde{v}_{1}, \ldots, \tilde{v}_{n(m)}\right)$ and $\left(\tilde{v}_{1}^{\prime}, \ldots, \tilde{v}_{n(m)}^{\prime}\right)$, and two permutations, $\pi$ and $\pi^{\prime}$, such that $\tilde{\alpha}_{m}\left(\pi \mid \tilde{v}_{1}, \ldots, \tilde{v}_{n}\right)>0$ and $\tilde{\alpha}_{m}\left(\pi^{\prime} \mid \tilde{v}_{1}^{\prime}, \ldots, \tilde{v}_{n}^{\prime}\right)>0$. One can choose arbitrarily small $\epsilon, \bar{\epsilon}>0$ such that for $m>m(t, \epsilon)$, the ratio between the weights $\frac{w_{j}\left(\tilde{\sigma}_{m}, \pi, \tilde{v}_{1}, \ldots, \tilde{v}_{n(m)}\right)}{w_{j}\left(\tilde{\sigma}_{m}, \pi^{\prime}, \tilde{v}_{1}^{\prime}, \ldots, \tilde{v}_{n(m)}^{\prime}\right)}$ that are given in (11) is arbitrarily close to 1 . This follows from the fact that in each round $t \leq j$ such that $\tilde{\sigma}_{m}^{t}>\epsilon$, the DM approaches candidates with a value $v>v_{h}-4 \bar{\epsilon}$. We can now apply an argument that is similar to the one we applied in the proof of Lemma 1 in order to show that for a sufficiently large $m$, it must be that $\sum_{j \in B_{t}} \sum_{r \in B_{t}} \frac{\lambda_{j r}\left(\tilde{\alpha}_{m} \tilde{\sigma}_{m}\right)}{\lambda_{j}\left(\tilde{\alpha}_{m} \tilde{\sigma}_{m}\right)}>k\left|B_{t}\right|$. This stands in contradiction to the fact that $\sum_{j \in B_{t}} \sum_{r \in B_{t}} \frac{\lambda_{j r}\left(\tilde{\alpha}_{m} \tilde{\sigma}_{m}\right)}{\lambda_{j}\left(\tilde{\alpha}_{m} \tilde{\sigma}_{m}\right)} \leq \sum_{j \in B_{t}} \sum_{r \in N} \frac{\lambda_{j r}\left(\tilde{\alpha}_{m} \tilde{\sigma}_{m}\right)}{\lambda_{j}\left(\tilde{\alpha}_{m} \tilde{\sigma}_{m}\right)}=k\left|B_{t}\right|$.

\section{Proof of the proposition}

By Lemma 4, there exists a number $T>0$ such that for every $n \in \mathbb{N},\left|T\left(\hat{\sigma}_{n}\right)\right|<T$. Let us choose a large $n^{\star}$ and consider a realisation in which there are more than $T+k$ candidates with a value $v>v^{\star}$ (the probability of such a realisation goes to 1 as $n$ goes to infinity). Consider the last round $t \in T\left(\hat{\sigma}_{n}\right)$. There must be at least $k+1$ candidates who are scheduled to receive an offer in rounds $\{t\} \cup N / T\left(\hat{\sigma}_{n}\right)$ and who each have a value greater than $v^{\star}$. The optimality of the DM's strategy requires that in round $t$, he make an offer to the candidate for whom $v p(v)$ is maximised (among the candidates who are scheduled to receive offers in rounds $\left.\{t\} \cup N / T\left(\hat{\sigma}_{n}\right)\right)$. By the assumption that $v p(v)$ is strictly decreasing for $v>v^{\star}$, there are at least $k$ candidates who are more valued than the candidate who is approached in round $t$. Therefore, $\hat{\sigma}_{n}^{t}>0$ cannot be a part of an SNE. 


\section{Proof of Proposition 3}

Consider the private offers regime and suppose that there exists an SNE such that $\gamma>0$. Let $t \in\{1, \ldots, n\}$. Recall that the DM's unique best response is to approach the candidates according to the order of their values. It follows that

$$
\begin{array}{r}
\operatorname{Pr}\left\{x_{i}=t-1, s_{i}=1, i \text { receives an offer }\right\}= \\
\int_{v_{l}}^{v_{h}} \ldots \int_{v_{l}}^{v_{h}} \mathbb{1}_{t-1}\left(v_{1}, \ldots, v_{n}\right)\left(1-\gamma p\left(v_{\pi_{1}\left(v_{1}, \ldots, v_{n}\right)}\right)\right) \ldots\left(1-\gamma p\left(v_{\pi_{t-1}\left(v_{1}, \ldots, v_{n}\right)}\right)\right) \\
p\left(v_{\pi_{t}\left(v_{1}, \ldots, v_{n}\right)}\right) f\left(v_{1}\right) \ldots f\left(v_{n}\right) d v_{1} \ldots d v_{n}
\end{array}
$$

where $\pi$ is a permutation such that $v_{\pi_{1}\left(v_{1}, \ldots, v_{n}\right)} \geq \ldots \geq v_{\pi_{n}\left(v_{1}, \ldots, v_{n}\right)}$ and $\mathbb{1}_{t-1}\left(v_{1}, \ldots, v_{n}\right) \in$ $\{0,1\}$ is an indicator that equals 1 if and only if $x_{i}=t-1$. Note that (12) is decreasing in $\gamma$ for $t \geq 2$. For $t=1$, (12) does not depend on $\gamma$. It follows that $\operatorname{Pr}\left\{x_{i}=0 \mid s_{i}=1, i\right.$ receives an offer $\}$ is increasing in $\gamma$. In words, when $\gamma$ is higher, receiving an offer is a stronger signal of being the most-valued candidate. It follows that if there exists an SNE in which $\gamma>0$, then there exists an SNE in which $\gamma=1$.

Fix $c^{\star}$. If there exists an SNE in which $\gamma=1$, then for $c<c^{\star}$ there exists an SNE in which $\gamma=1$. If there exists no SNE in which $\gamma=1$, then for $c>c^{\star}$, there exists no SNE in which $\gamma>0$. An SNE in which $\gamma=1$ and the DM approaches the candidates according to their values induces the highest possible payoff for the DM and an SNE in which $\sigma=0$ induces the lowest possible payoff for the DM. 